\title{
Examining Instructor Use of Learning Analytics
}

Suzanne L. Dazo

Nicholas R. Stepanek

Aarjav Chauhan

Brian Dorn

University of Nebraska Omaha

Omaha, NE 68182, USA

sdazo@unomaha.edu

nstepanek@unomaha.edu

achauhan@unomaha.edu

bdorn@unomaha.edu

\begin{abstract}
This study takes an instructor-centric approach to Learning Analytic (LA) research by analyzing instructor use of the LA within an educational streaming video platform called TrACE. The goal of this study is to understand how instructors naturally interact with analytic dashboards through an empirical analysis. To accomplish this, data of 14 instructors from three institutions that used TrACE from Spring 2015 to Spring 2016 was collected. Data was analyzed to identify frequency of analytic visits, duration of analytic use, differences in analytic use, and differences in use between semesters. Instructors demonstrated preferences for some analytics over others, but the majority of teachers generate short sessions that may not allow for in-depth exploration in analytics. Finally, instructor activity is not always consistent between semesters. Focus groups were conducted to explore motivations behind these findings and future work includes developing LA that address discovered issues.
\end{abstract}

\section{Author Keywords}

analytics usage trends; instructor support

\section{ACM Classification Keywords}

H.5.2 [Information interfaces and presentation (e.g., $\mathrm{HCl}$ )]: User Interfaces 


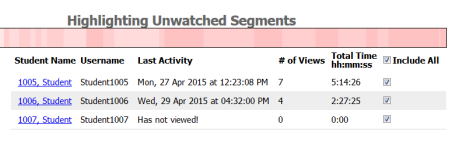

Figure 1: Media Activity informs instructors of various student viewing behaviors in a table

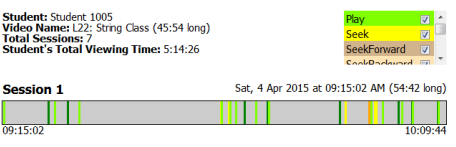

Figure 2: Session Summaries gives details on actions taken each time a student watched a video in the form of timelines

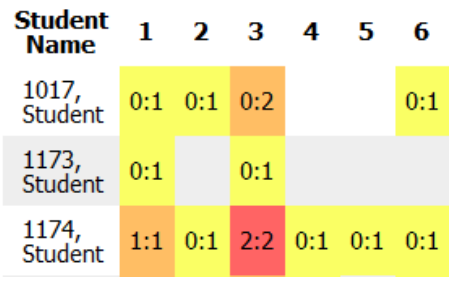

Figure 3: Annotation Summary is an example of an analytic with a heatmap that becomes more red as a student posts more

\section{Introduction}

Learning Analytics (LA) is the measurement, collection, analysis and reporting of data about learners and their contexts [8]. LA can be used among various stakeholders (students, educators, administrators) and can vary in scope from the course-level all the way up to broader contexts involving multiple courses and institutions. Instructors, who play an integral role in the classroom, are necessary for the effective use of these analytics.

The value of instructors' use of LA has also been emphasized in previous work. Scheffel [15] stressed the importance of supporting teacher reflection and awareness. This includes the need for teachers to be aware of student activity, mastery, and mistakes in a way that can also support reflection [16]. Also important to instructors is the ability to respond to individual students and adapt their teaching to address the challenges identified through LA. Reflective practice [17] encourages instructors to consider the goals important to them in their course contexts, gather data, and process that data to accomplish or redefine those goals. Supporting reflective practice for instructors is important early in the design of an LA, otherwise they may not intervene when LA indicate that a student is struggling or may not change their methods when analytics indicate clear issues which should be addressed [18]

The Learning Analytics Cycle [4] is influenced by reflective practice and describes how analytics should process and present information to be most useful. The cycle includes an intervention phase, in which instructors take specific actions that have an effect on learners (or even supporting reflective practice that influences teaching in future semesters). Current LA practices do not necessarily support teacher reflective practice due to collecting data that may not be directly based on what instructors want to know.
Current research has given us excellent evidence on what qualities can make for effective analytics [15] and many tools exist with the intention of informing instructors about student activities (see e.g., [5, 14]). However, Dyckhoff et al. identified that of almost 30 LA analyzed, they regularly do not help annswer all the questions teachers have about classes [7]. Additionally, instructors report challenges interpreting visualizations which are often created with the assumption that users are familiar with data mining techniques and complex analysis methods [16]. There is a need to further consider the pedagogical issues involved, and how to best facilitate the interaction between instructors and students [3]. Some setbacks include a gap in studies on the entire course context [9], a lack of time in development to involve teachers, and a lack of existing community that prioritizes the involvement of instructors in design [13].

In moving forward, we propose that one way to address these issues is with a instructor-centered approach that seeks to understand how teachers are actually using LA for their teaching interventions in course settings. Thus, the purpose of this study is to move toward improving LA design by providing a better understanding of how instructors use LA in the field. In this work, we empirically investigate how 14 university instructors currently interact with an analytics dashboard as part of their normal teaching duties.

\section{Study Platform Description}

For our study, we look at instructor use of analytics in TrACE an online video-playback platform that was developed with the goal of supporting collaboration among students within university courses making heavy use of web-based video (ie., online and blended courses) [6]. Instructors upload videos for students to watch, and students can annotate and reply to annotations in videos. As a part of this system, an initial LA suite was developed to allow for instructors to interpret student viewing behaviors, which was a need iden- 


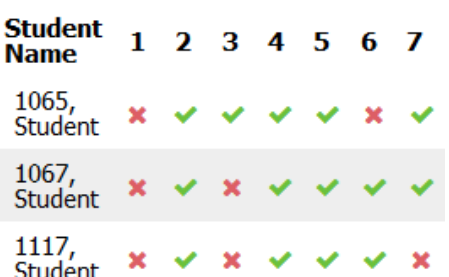

Figure 4: Viewing Summary answers "did my student access this video?" with a check or $X$

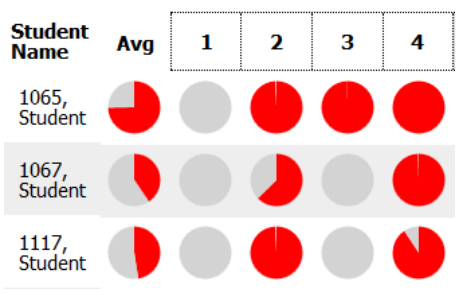

Figure 5: Percentage Viewed has a pie chart to show how much of a video was watched tified among several teachers and education researchers $[11,10]$. Observing instructor use of TrACE is useful as video elements are widely used in large-scale online education platforms (e.g. Coursera, Udacity, Khan Academy), but as a research prototype, it can serve as a more flexible platform that is responsive to instructor needs on a smaller scale during development. Also, this tool's LA have common quantitative measures of broad interest such as view count, percent of content viewed, number of posts, time spent, and when content was viewed.

To better understand these analytics, we describe what questions instructors can answer and the visualization used. Screenshots of selected analytics are provided. Most analytics show video level detail for a single course and display activity for all students enrolled.

Media Activity (fig. 1) Answers how often, what parts, and when students watch a single video. It also shows how much time students spent a particular video. Timestamps and view counts are visualized using a heatmap table for what parts of the video were watched (or not) by selected groups of students.

Session Summary (fig. 2) (accessed via Media Activity) This also answers how long, how often and what actions were taken in greater detail for a single student. It is presented as a list of session timelines (from opening the video to leaving the video page) with color-coded markers depicting what actions occurred when.

Annotation Summary (fig. 3) Answers how many posts and replies did students make presented as a global distribution heatmap of relative activity by quartile.

Loyalty - Answers how many times students opened a video also presented as a global course heatmap.

Recency - Answers when was the last time students interacted with the video in terms of days and color-coded by four increasing buckets of time.
Viewing Summary (fig. 4)- A check (yes) or X (no) answering if students opened the video.

Percentage Viewed (fig. 5)- A pie chart showing what \% of the video students watched.

View Count graph - A histogram showing how the number of views differ (between students, between videos, over the semester).

\section{Methods}

To understand how instructors currently interact with LA, we analyzed usage data of 14 instructors that used TrACE's LA from January 2015 to May 2016 (Spring '15 to Spring '16 semesters in North American vernacular). Most of these instructors had used TrACE prior to this analysis, so they may have already formed habits. This was a key difference from many other studies on system use, which were tested with first-time users of LA $[2,12,1]$. The largest class had 59 students. There was a mix of undergraduate and graduate courses, the majority being in STEM disciplines with a few education and political science courses. On average, instructors taught 1.8 courses per semester with a maximum of 3 courses in any given semester Some instructors reused videos in multiple sections of classes. Nearly all courses were either online or flipped/blended courses.

During the study, instructors were given a tutorial on using TrACE each semester, but otherwise used the system freely. Logfile data collected includes which analytics were accessed, who accessed them, timestamps, and other action details (such as applying filters or closing reports). Frequency and duration of analytic use were calculated for each instructor. Sessions less than one second were filtered out as they were likely misclicks. After filtering, one instructor had no data and was excluded. Duration data were not evenly distributed among teachers, so medians and non-parametric tests were used. Frequency and median duration were compared to determine analytic prefer- 


\begin{tabular}{|c|lll|}
\hline & Sp15 & Fa15 & Sp16 \\
\hline 1 & 0.35 & 0.67 & 0.33 \\
\hline 2 & $\mathbf{1 0 . 3}$ & $\mathbf{5 . 3}$ & $\mathbf{4 . 4}$ \\
\hline 3 & 0.22 & & 0.22 \\
\hline 4 & $\mathbf{1 . 2 7}$ & $\mathbf{2 . 5 6}$ & $\mathbf{1 . 0 3}$ \\
\hline 5 & $\mathbf{1 . 9 7}$ & $\mathbf{1 . 3 7}$ & 0.61 \\
\hline 6 & 0.21 & & 0.02 \\
\hline 7 & 0.26 & 0.14 & 0.14 \\
\hline 8 & 0.74 & & \\
\hline 9 & 0.27 & 0.81 & \\
\hline 10 & & & 0.29 \\
\hline 11 & & & $\mathbf{1 . 5}$ \\
\hline 12 & & 0.77 & \\
\hline 13 & & 0.09 & \\
\hline
\end{tabular}

Table 1: Frequency of overall analytic use normalized by number of videos in all courses

\begin{tabular}{|c|lll|}
\hline & Sp15 & Fa15 & Sp16 \\
\hline $\mathbf{1}$ & 6 & 18.5 & 42.5 \\
\hline 2 & 19 & 17 & 20 \\
\hline 3 & 52 & & 24 \\
\hline $\mathbf{4}$ & 42.5 & 61 & 58 \\
\hline $\mathbf{5}$ & 35 & 28.5 & 83.5 \\
\hline 6 & 31 & & 35 \\
\hline $\mathbf{7}$ & 8.5 & 33 & 38 \\
\hline 8 & 11 & & \\
\hline 9 & 35 & 44 & \\
\hline 10 & & & 35.5 \\
\hline 11 & \multicolumn{3}{|c|}{27} \\
\hline 12 & \multicolumn{3}{|c}{ N/A } \\
\hline 13 & \multicolumn{3}{|c}{} \\
\hline
\end{tabular}

Table 2: Median duration (in seconds) instructors spent in all analytics. Instructors with a significant difference in duration between semesters $(p<0.05$ using a Kruskal-Wallis test) are in bold ence. Also, visit frequency was compared across semesters to understand if interaction was consistent over time. Frequency was calculated both in a raw form and as a Visit to Videos ratio because an instructor may not be teaching the same courses or using the same videos every semester.

\section{Results}

The remaining 13 instructors visited the LA 48 times in a semester on average, although this varied between instructors ( 1 to $200+$ visits). TrACE's LA were visited 1268 times overall with a distribution of 494 sessions in Spring '15, 410 sessions in Fall '15, and 364 sessions in Spring '16. Table 1 presents how often an instructor visited any analytic normalized by the number of videos in their courses. Analytic visits at a ratio greater than once per video are in bold. $69.3 \%$ of instructors did not visit any analytic at least once per video, so many may be viewing multiple videos for each visit, or not viewing analytics for some videos at all.

Table 2 shows the median time instructors spent in any of the analytics each semester. Median time spent varied widely (6s to $83.5 \mathrm{~s}$ ), but most instructors do not spend much time with analytics. Instructors 4 and 11 stand out for having spent more time in LA while also visiting at least once per video. These users may have a habit of analyzing student behaviors in every video. Instructor 5 had the highest median of 83.5 seconds but only viewed analytics 0.61 times for every video. One possible explanation may be that this instructor may be analyzing multiple videos at once.

In most cases, instructors did not have a significant difference in duration between analytics within a semester This could be either because there were not many visits to the analytics or there was no preference. Excluding two instructors who only used one analytic, 4 of the 11 instructors showed a significant preference for an analytic in at least one semester. These instructors are in- structor $2\left(X^{2}(7)=41.1, p<0.001\right)$ in Spring '15, instructor $3\left(X^{2}(2)=9.681, p=0.008\right)$ in Spring '15, instructor 4 in Fall '15 $\left(X^{2}(7)=34.3, p<0.001\right)$ and Spring '16 $\left(X^{2}(7)=14.67, p=0.04\right)$, and instructor 11 $\left(X^{2}(6)=15.7, p=0.015\right)$ in Spring '16

We ranked the analytics by the mean usage (as duration) and calculated how many instructors used that analytic the most. Media Activity (5), Percentage Viewed (3), Recency (2), Annotation Summary (2), and View Count Graph were used the most. Some of these instructors are not only dedicating more time to understanding these analytics, but they visit them more frequently. The opposite of this is instructor 2, who used analytics like View Count Graph (10 visits) and Recency (6 visits) longer than the other analytics, but did not visit those analytics the most often (avg $=11.6$ visits per analytic). It could mean that these analytics take more time to parse than the others or are only needed occasionally.

Finally, to answer if instructors are interacting with analytics differently between semesters, we compared frequency of analytic visits (Table 1) and median duration with a KruskalWallis test. No instructor that started visiting infrequently increased this proportion to be one visit per video and instructors who visited often continued to do so. From Fall ' 15 to Spring '15, instructors visited analytics either the same amount or less per video. In general, many instructors were spending more time in the analytics but visiting less often. Instructors could be doing in-depth analyses or the analytics may take more time to interpret. We were mindful of this when interviewing educators about their analytic use.

\section{Discussion and Follow-up}

From our results, we noticed a few key takeaways on how instructors used LA. First, frequency was often less than one visit per video, although a few instructors stood out as visiting more often. Duration was surprisingly low univer- 
Quote 1: "In general, initially I was very hungry for analytics. Over time I realized that these analytics, I have the data but I don't necessarily know what interventions to use to get the end result that I want."

Quote 2: "The biggest change I made was moving from only having video watching to requiring comments as a way to formatively assess my class."

Quote 3: 'You're telling me that a student has opened the video, watched a second of it. That means nothing to me. If the Viewing Summary was like a combination between things like Percentage Viewed and Recency, this would be the only thing that I really would need."

Figure 6: Highlighted quotes from instructor focus groups. sally and whether or not instructors demonstrated spending more time in some analytics over others was mixed. Surprisingly, those who overcame the hurdles of initial adoption did not seem to use LA more. Frequency of visits decreased between semesters, such as from Fall ' 15 to Spring ' 15 , but half of instructors also spent more time with LA.

We present focus group data somewhat informally as a follow-up to explore possible reasons behind these findings. Focus groups with six instructors from the above study were conducted in two sessions ( 3 each). Four of the instructors used the system for multiple semesters and four instructors also taught in STEM disciplines. We had a mix of instructors who might informally be categorized as high analytics users (2), nonusers (1), and somewhere in the middle (3). An affinity diagram was created from transcripts, and a bottom-up approach was used to develop themes. Overall, instructors described shifting away from analytics in favor of other methods, course contexts affecting analytic use, and why some analytics were preferred.

A common issue brought up was the challenge of interpreting data. When one instructor first started using TrACE several semesters ago, s/he was excited for analytics and all that could be analyzed, but came to realize that the information was not presented in an actionable way. As a result, the instructor shifted instead to reading student posts, which allowed for easier understanding of student progress (Quote 1, fig. 6). Many other instructors said they focused on student activities at first but in the face of difficulties switched to reading posts. Another reason for reading posts was that instructors were not as confident that the analytics captured true student engagement with the material.

Instructors reported having to jump between analytics and remember information in order understand student activities. Putting all of the analytics for one student in one place would allow for a view of a single student without being distracted by other data. Also, it is cumbersome to search for a student's post, so an analytic that collects students qualitative information in one place would also be useful.

Some instructors changed their use of the system to allow for more formative assessment. Formative assessment involves informal assessments to gauge where students are in their learning and change teaching accordingly. For some, the quantitative data in the analytics did not always allow for this (Quote 2, fig. 6). With this instructor as an example, comments offered a faster way to determine what student misconceptions were so that they could be addressed directly in class.

In general, instructors reported that they often refer to the analytics or read student posts in order to gauge what material or questions should be addressed in class. These instructors read through student questions or responses to instructor-created prompts to determine where extra time should be spent reviewing. Even a nonuser of analytics was very active in the posting aspects of TrACE and was more interested in using analytics for post-semester review than as a way to receive information on current behavior.

How the instructors use videos within their class affects which analytics they want and how often those analytics are used. Instructors using videos in-class did not use the analytics as often as those with lecture videos used outside of class. Instructors with in-class activities did not need analytics to gauge student preparation and participation, as preparing by watching videos before class was not a part of the course context. Some instructors had multiple videos every lecture, while others only used them infrequently (such as for lab activities) and would cease to use TrACE at or before midterms. The instructors with weekly lecture videos were very quick to self-report specific analytics that 


\section{(c)}

Watch $12 \%$ of the video before

Fri, Sep 302016 1:54pm

Figure 7: Part of the LA created to support instructor needs. Students see their progress towards the expectations an instructor created earlier. The watching expectation for this video is complete. they used to understand their class, and often stated that they used analytics at least once a week when the weekly video was due. These instructors were much more reliant on analytics because of their course context(weekly videos and participation grades determined by analytics). There were some instructors that simply did not have any participation requirement, and thus rarely checked analytics.

In asking instructors what analytics they choose to use and why, Percentage Viewed, Annotation Summary, and Recency were popular analytics. Percentage viewed was mentioned by almost all instructors. A pie chart provided a visual indicator and was easier interpret than a table of numbers. One instructor reported that s/he only used Viewing Summary and Percentage viewed, as knowing in general if students were watching was enough information. Another instructor used Percentage Viewed as the Viewing Summary was misleading for their goal to quickly assess if students watched a video (Quote 3, fig. 6).

A heavy user of the system often mentioned Percentage Viewed and Annotation Summary because the participation grade for the class was based on video viewing and collaboration(as indicated by \# of posts). Another instructor did not initially care much for posting, but a desire for more formative assessment in the class led to increased interest in Annotation Summary and reading student posts.

Although a significant amount of time was spent in Media Activity compared to other analytics, not many instructors mentioned it as a primary way to learn about their class. This analytic is very detailed and can be complex to interpret. Instructors sometimes used it to explore at-risk students as identified by other analytics instead of as a primary source of data because of its complexity.

\section{Ongoing and Future Work}

Creating effective LA is an ongoing topic in education research. The contribution of this work is an insight into how and why instructors use LA in certain ways. Instructors are very limited in the time that they spend in analytics, so even minor challenges such as context switching, mental calculation, or even unexpected course changes become a huge barrier to LA use. Instructors want to use LA related to goals, but some goals are not quantitative. Some instructors look at quality of student understanding, where numerical analysis may not be as useful.

In our follow-up work based on these results, we have designed a new set of LA in TrACE that use instructors' individual expectations to tailor analytic feedback. Instructors wanted to model their expectations for how students should use TrACE, view student progress in aggregate, and allow for students to also see their progress in meeting these expectations (fig. 7). Such expectation-based analytics aggregate data from multiple sources. Instead of looking at multiple analytics, a single analytic can combine multiple data sources into one view. This way, instructors would not be burdened with mental calculations to determine whether a not a student met an expectation.

A pilot study with these new tools was conducted in the Fall '16 term and analysis is ongoing. In addition to future studies on system usability, we also look to understand if the presence of this expectation-oriented tools affects instructor intervention, LA use, or student performance.

\section{Acknowledgements}

This work is funded in part by the National Science Foundation under grant IIS-1318345. Any opinions, findings, and conclusions, or recommendations expressed in this materia are those of the authors and do not necessarily reflect the views of the NSF. 


\section{References}

[1] Liaqat Ali, Marek Hatala, Dragan Gašević, and Jelena Jovanović. 2012. A qualitative evaluation of evolution of a learning analytics tool. Computers \& Education 58, 1 (2012), 470-489.

[2] Kimberly E Arnold and Matthew D Pistilli. 2012. Course signals at Purdue: using learning analytics to increase student success. In Proc. LAK'12. 267-270.

[3] Mohamed Amine Chatti, Anna Lea Dyckhoff, Ulrik Schroeder, and Hendrik Thüs. 2012. A reference model for learning analytics. Int. J. of Technology Enhanced Learning 4, 5-6 (2012), 318-331.

[4] Doug Clow. 2012. The learning analytics cycle: closing the loop effectively. In Proc. LAK'12. 134-138.

[5] Shane Dawson, Aneesha Bakharia, and Elizabeth Heathcote. 2010. SNAPP: Realising the affordances of real-time SNA within networked learning environments. In Proc. of the 7th Int. Conf. on Networked Learning. 125-133.

6] Brian Dorn, Larissa B Schroeder, and Adam Stankiewicz. 2015. Piloting TrACE: Exploring spatiotemporal anchored collaboration in asynchronous learning. In Proc. CSCW'15. 393-403.

[7] Anna Lea Dyckhoff, Vlatko Lukarov, Arham Muslim, Mohamed Amine Chatti, and Ulrik Schroeder. 2013. Supporting action research with learning analytics. In Proc. LAK'13. 220-229.

[8] Rebecca Ferguson. 2012. Learning analytics: drivers, developments and challenges. Int. J. of Technology Enhanced Learning 4, 5-6 (2012), 304-317.

[9] Wolfgang Greller and Hendrik Drachsler. 2012. Translating Learning into Numbers: A Generic Framework for Learning Analytics. Educational technology \& society 15, 3 (2012), 42-57.
[10] Lisa L. Lacher and Mark C. Lewis. 2015. The Effectiveness of Video Quizzes in a Flipped Class. In Proc. SIGCSE'15. 224-228.

[11] Mary Lou Maher, Celine Latulipe, Heather Lipford, and Audrey Rorrer. 2015. Flipped Classroom Strategies for CS Education. In Proc. SIGCSE'15. 218-223.

[12] Arham Muslim, Mohamed Amine Chatti, Tanmaya Mahapatra, and Ulrik Schroeder. 2016. A rule-based indicator definition tool for personalized learning analytics. In Proc. LAK'16. 264-273.

[13] Tamara Holmlund Nelson, David Slavit, Mart Perkins, and Tom Hathorn. 2008. A Culture of Collaborative Inquiry: Learning to Develop and Support Professional Learning Communities. Teachers College Record 110, 6 (2008), 1269-1303

[14] Cristóbal Romero, José Raúl Romero, Jose María Luna, and Sebastián Ventura. 2010. Mining rare association rules from e-learning data. In Proc. EDM'10. 171-180.

[15] Maren Scheffel, Hendrik Drachsler, Slavi Stoyanov, and Marcus Specht. 2014. Quality indicators for learning analytics. J. of Educational Technology \& Society 17, 4 (2014), 117-132.

[16] Maren Scheffel, Katja Niemann, Abelardo Pardo, Derick Leony, Martin Friedrich, Kerstin Schmidt, Martin Wolpers, and Carlos Delgado Kloos. 2011. Usage pattern recognition in student activities. In Towards Ubiquitous Learning. Springer, 341-355.

[17] Donald Schön. 1987. Educating the reflective practitioner. Josey-Bass Publishers, San Francisco, CA.

[18] Georgea M Sparks-Langer, Joanne M Simmons, Marvin Pasch, Amy Colton, and Alane Starko. 1990. Reflective pedagogical thinking: How can we promote it and measure it? J. of teacher education 41, 5 (1990), 23-32. 